\title{
Ultrafast Carrier Dynamics in Few-Layer Colloidal Molybdenum Disulfide Probed by Broadband Transient Absorption Spectroscopy
}

\author{
Pieter Schiettecatte, 1,2 Pieter Geiregat, 1,2 Zeger Hens 1,2
}

${ }^{1}$ Physics and Chemistry of Nanostructures, Ghent University, Belgium

${ }^{2}$ Center for Nano and Biophotonics, Ghent University, Belgium

\section{Introduction}

Insights into the photophysics of TMDs made by exfoliation or CVD have been pivotal for the advancement toward a broad range of applications

Colloidal synthesis has emerged as an inexpensive, scalable and highly-tunable alternative to exfoliated and CVD-grown TMDs

If colloidal methods have to become a true alternative, they should be benchmarked relative to CVD-grown and

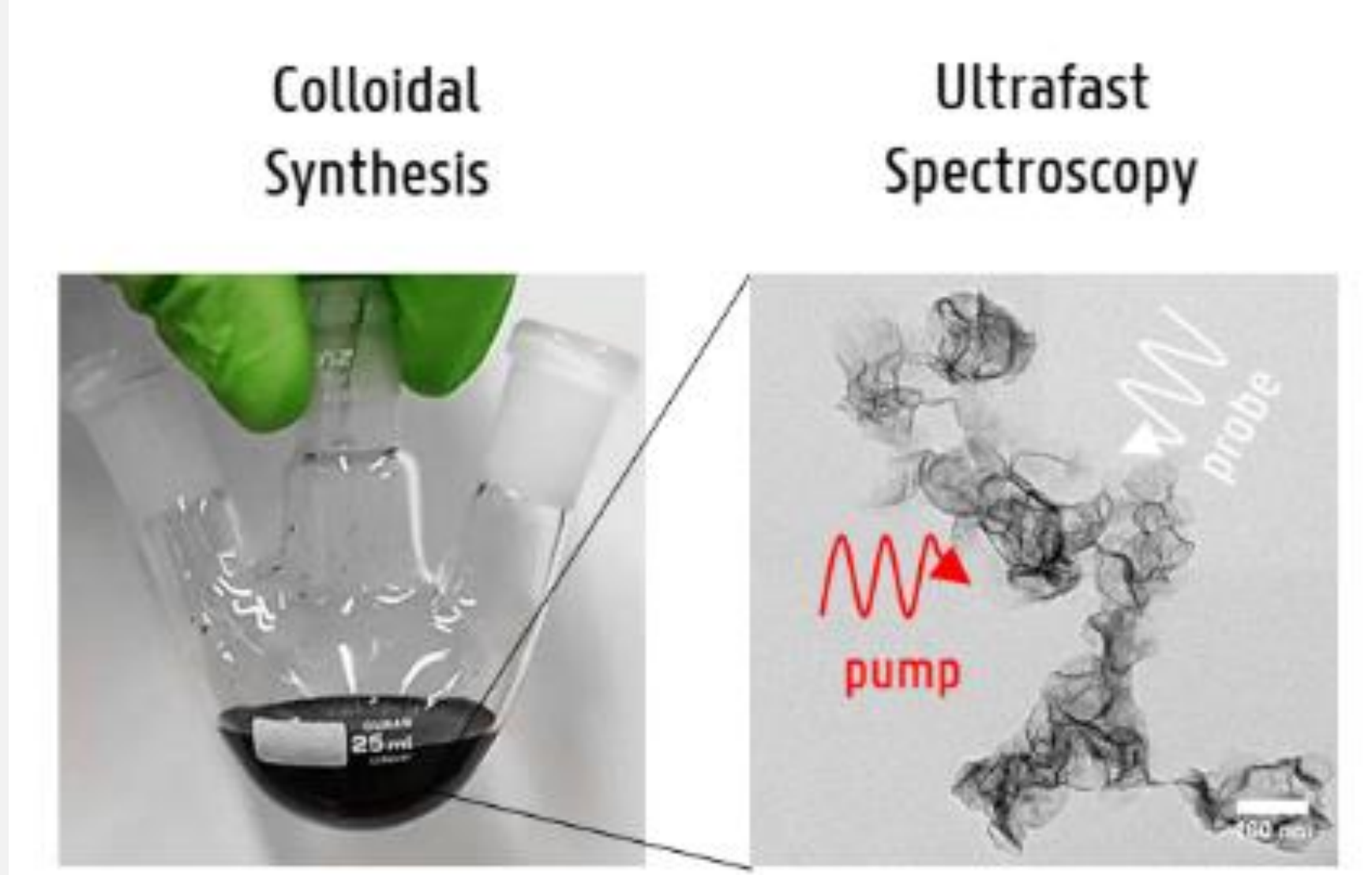

Charge Carrier Relaxation fast hole capture exfoliated TMDs

Colloidal Synthesis of Molybdenum Disulfide

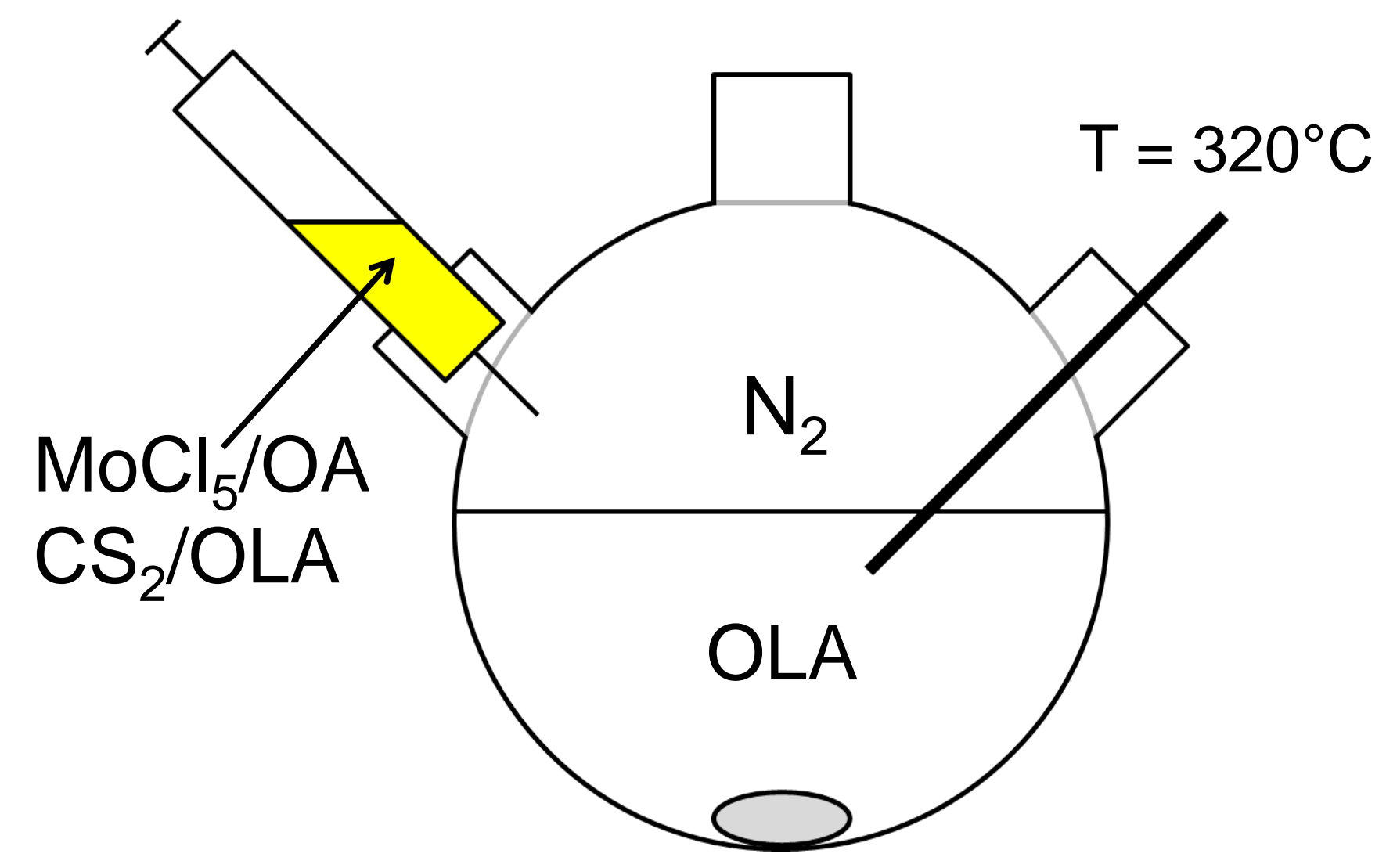

bottom-up synthesis of $\mathrm{MoS}_{2}$

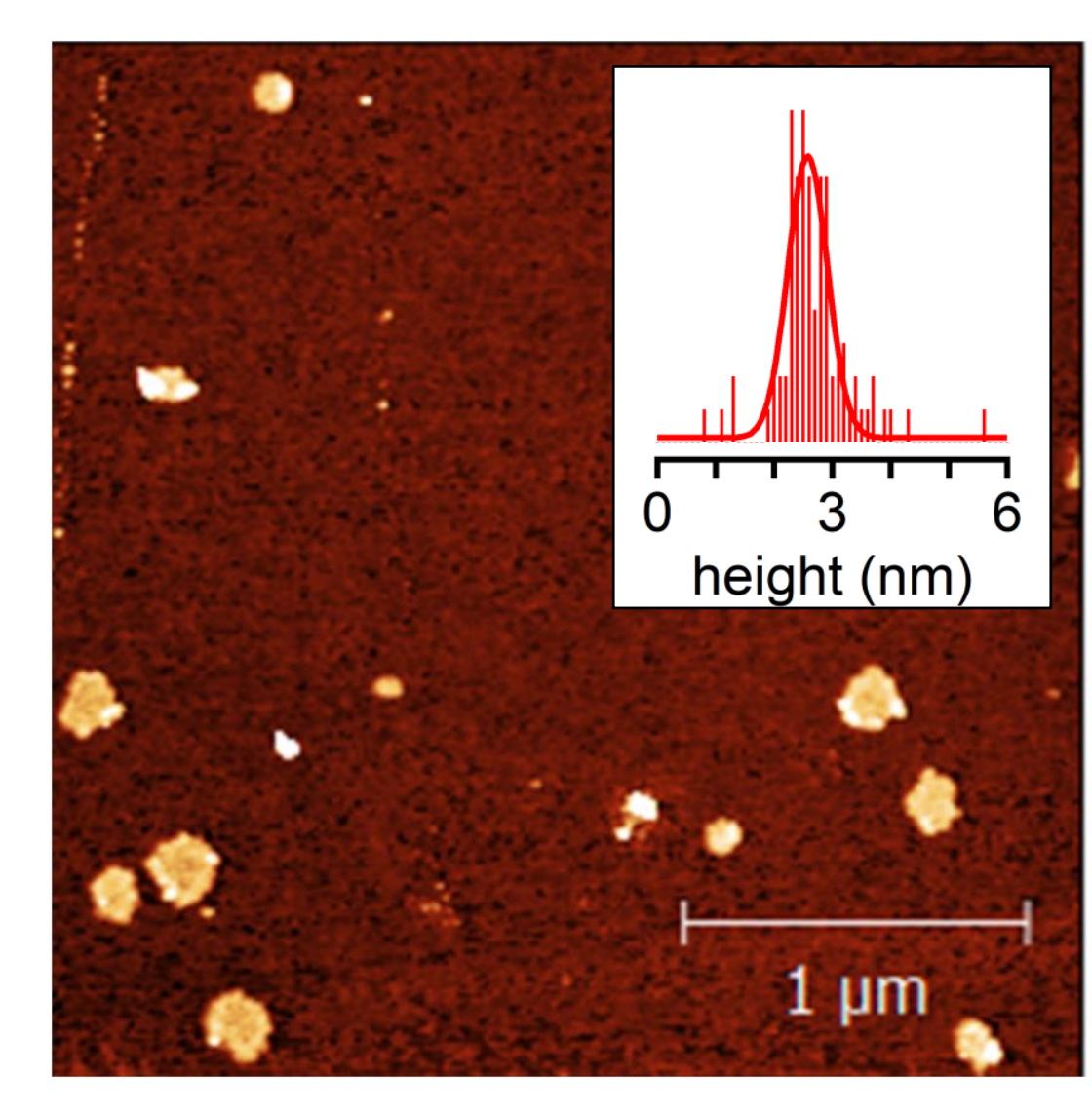

few-layered (AFM)

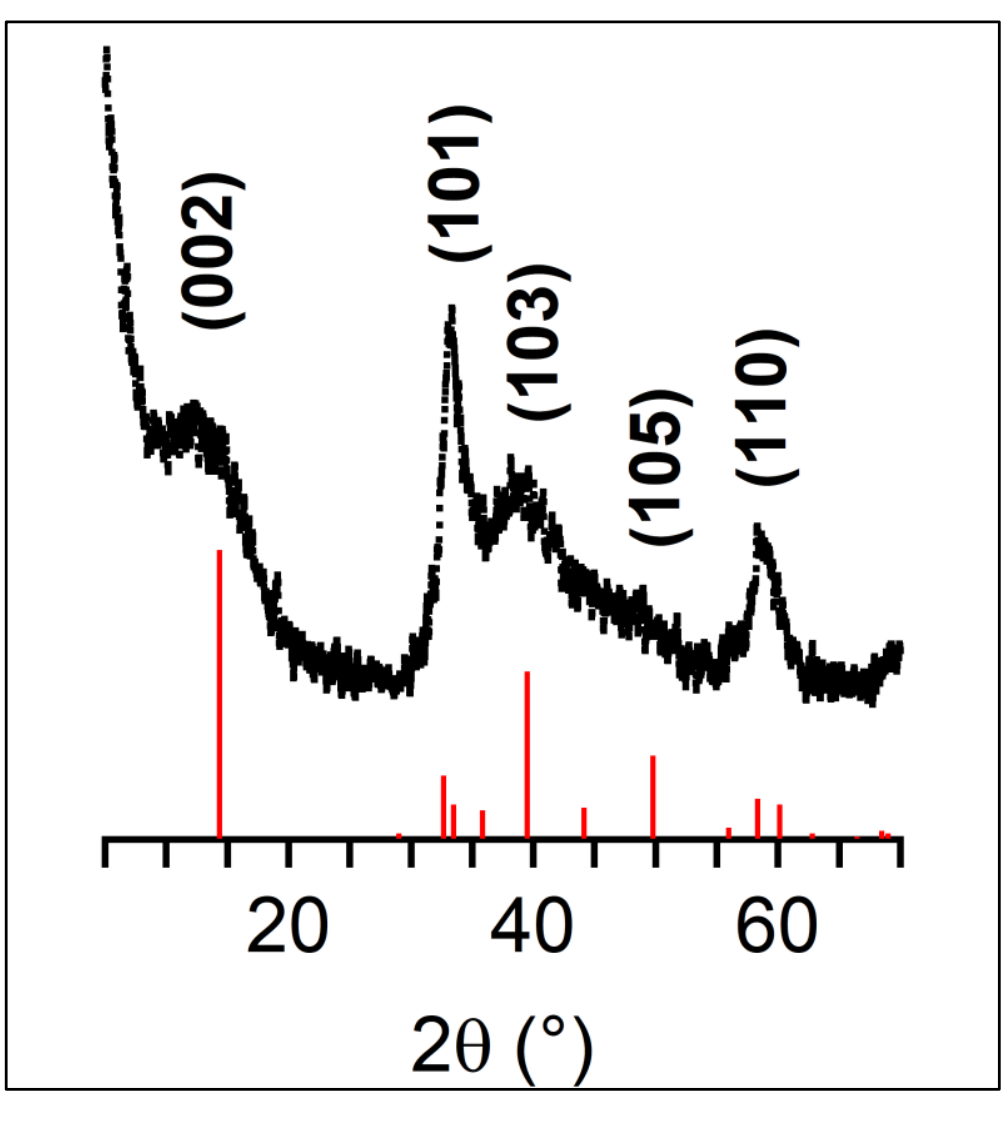

$2 \mathrm{H} \mathrm{MoS}_{2}$ (XRD and UV-vis)

The synthesis yields few-layered colloidal $2 \mathrm{H} \mathrm{MoS}_{2}$ nanosheets

Transient Absorption - Exciton Dynamics

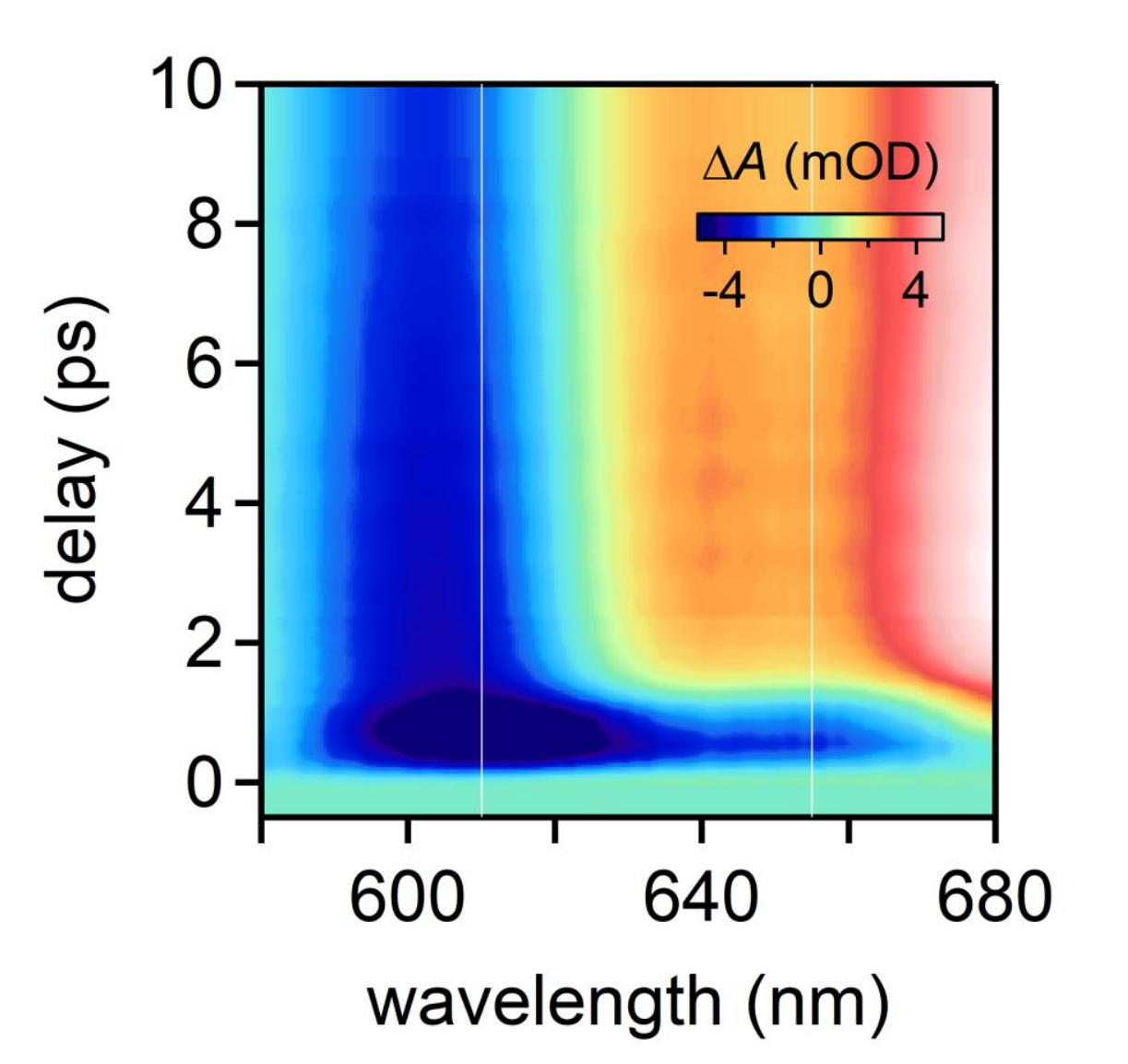

short-living bleaches around band-gap

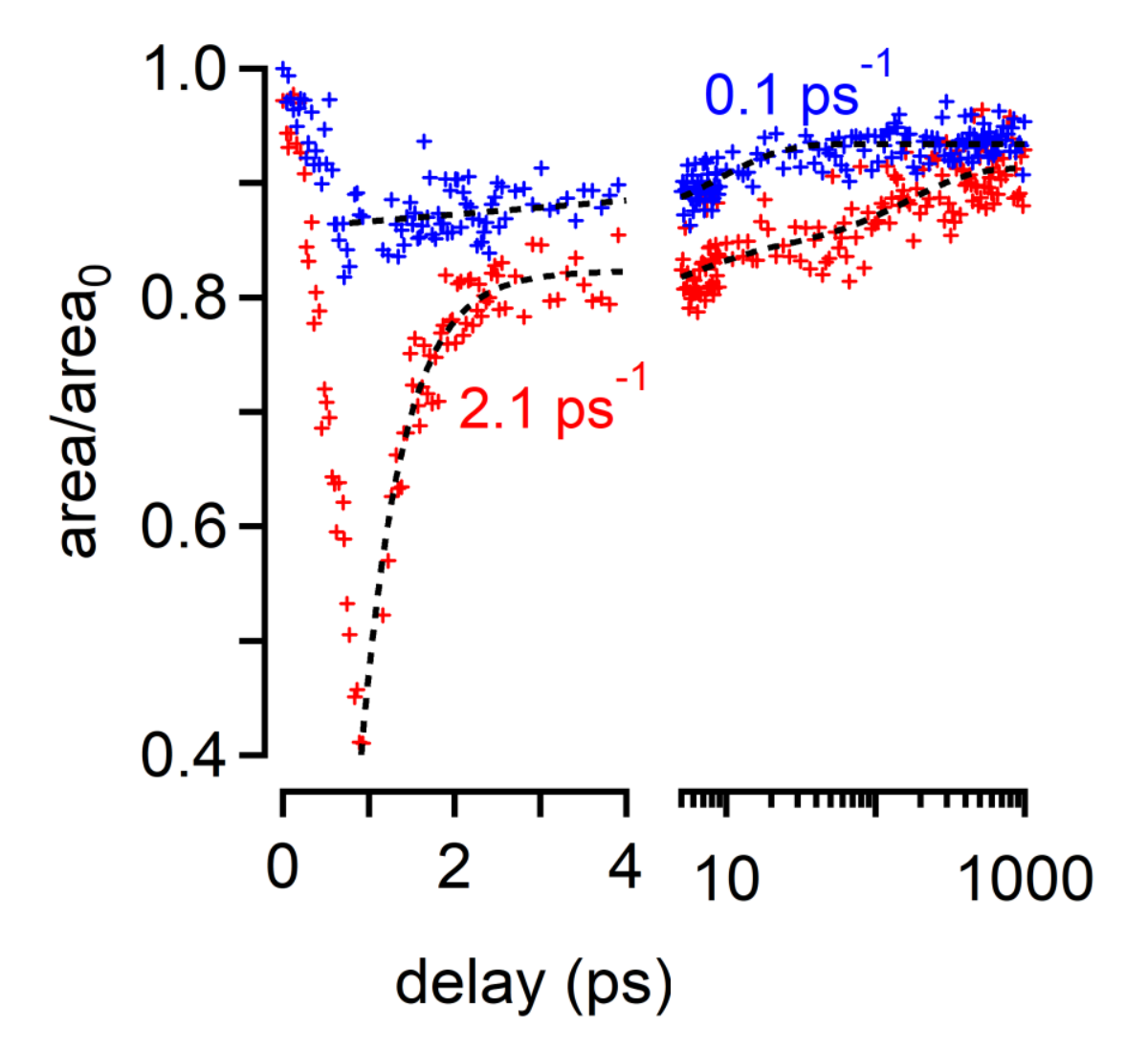

netto bleaches due to state filling rapid decay of $A$ exciton bleach

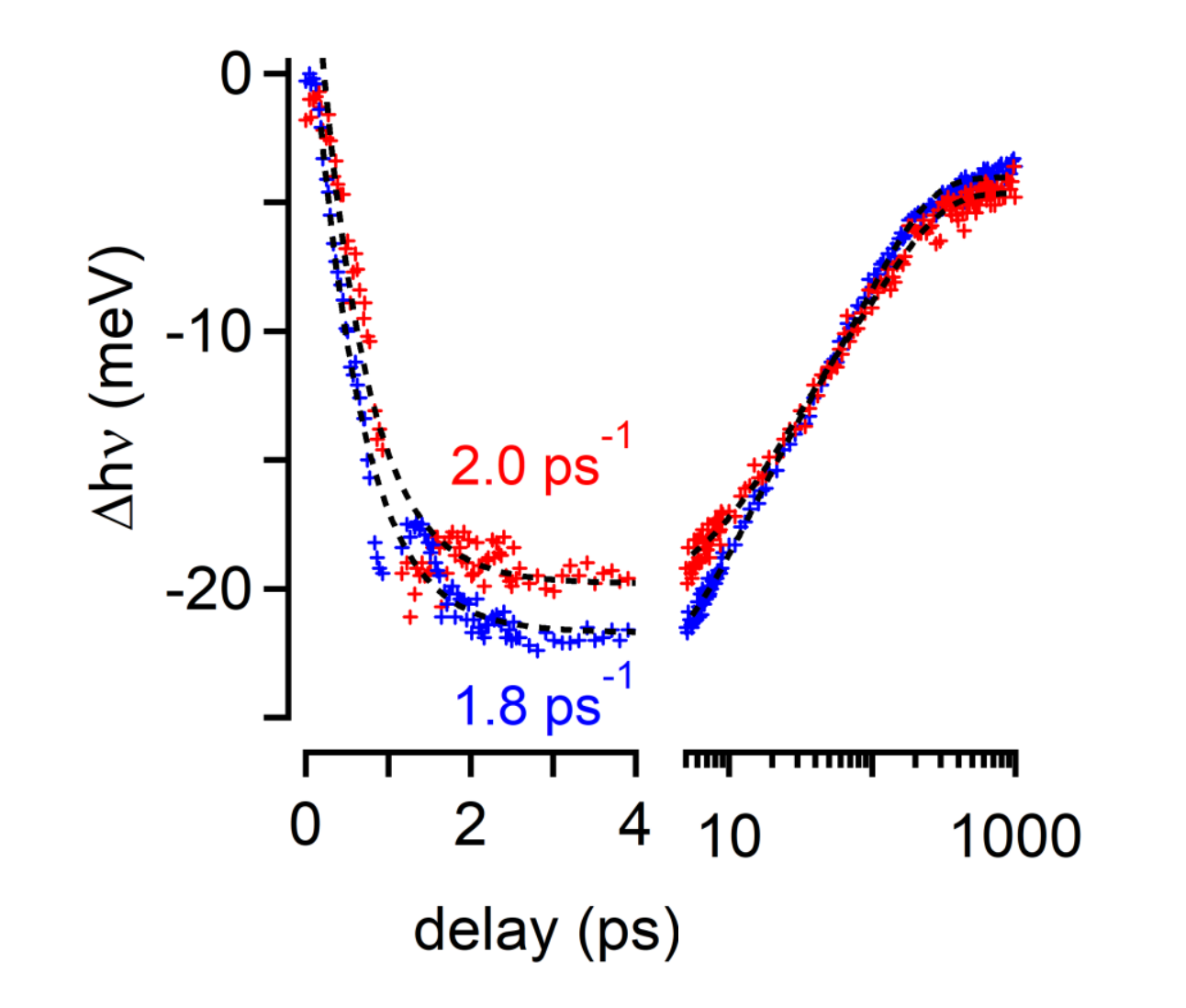

dynamics of the spectral shift and broadening happen on a remarkably similar time scale

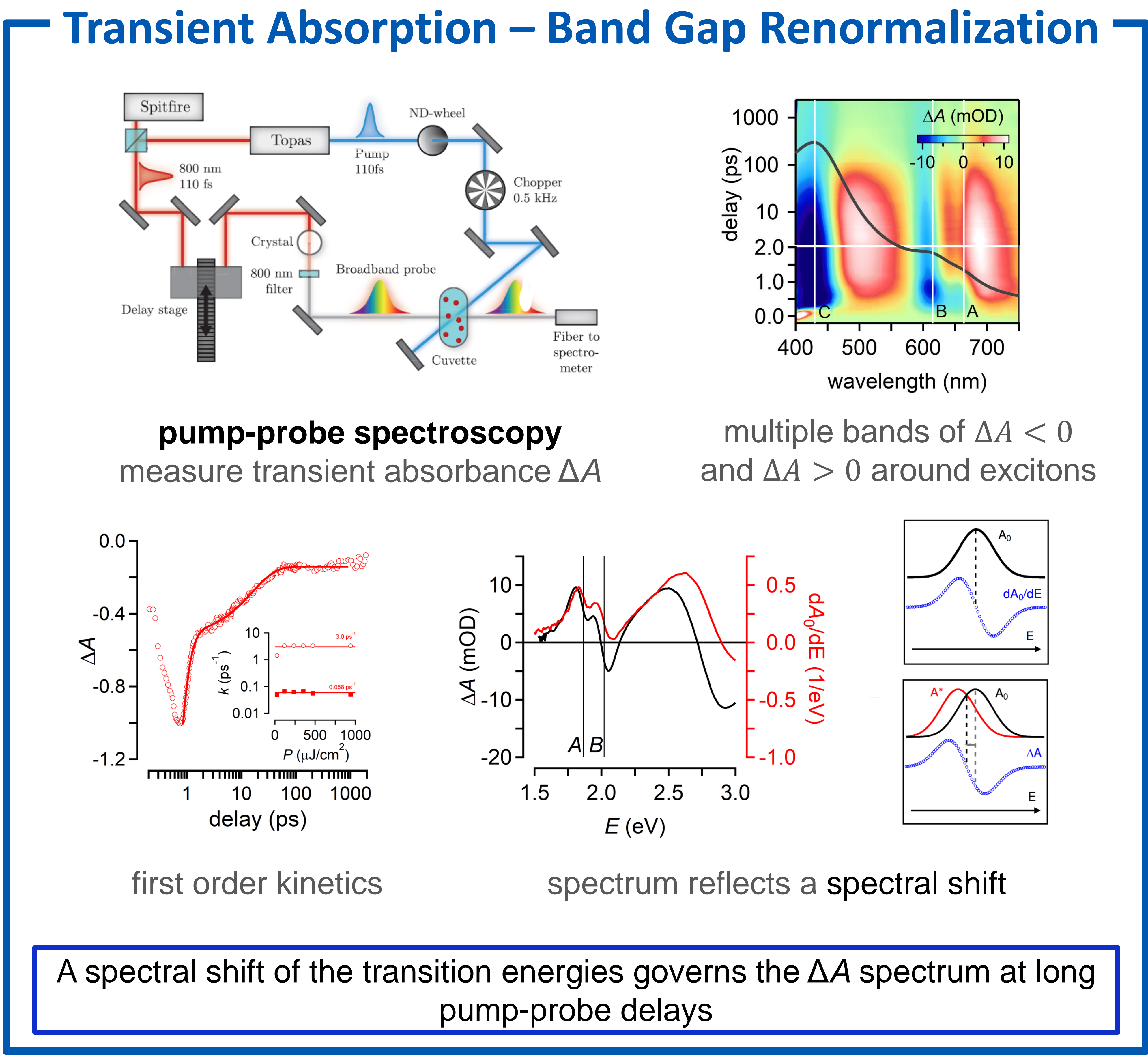

Transient Absorption - Intraband Absorption

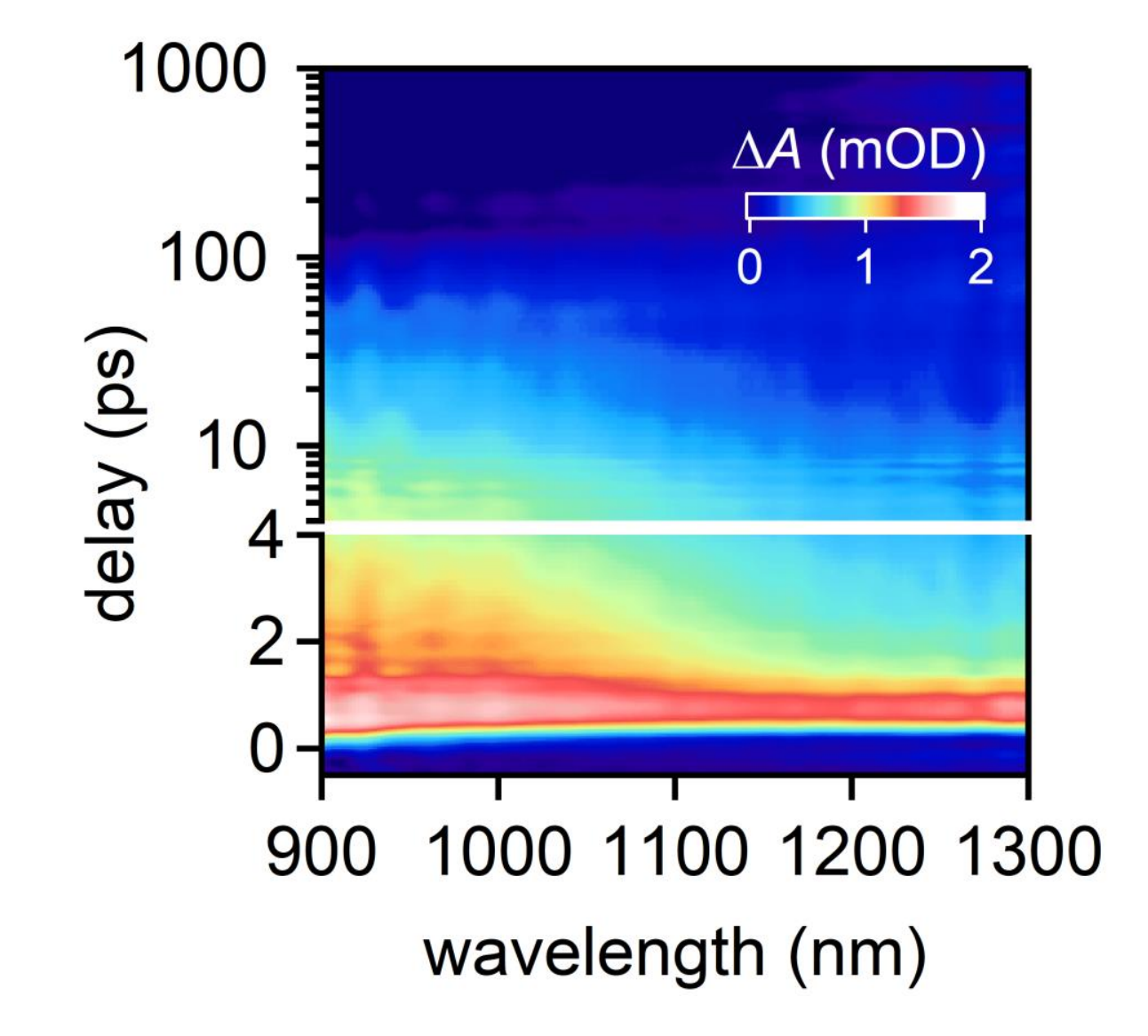

photoinduced absorption related to intraband transitions

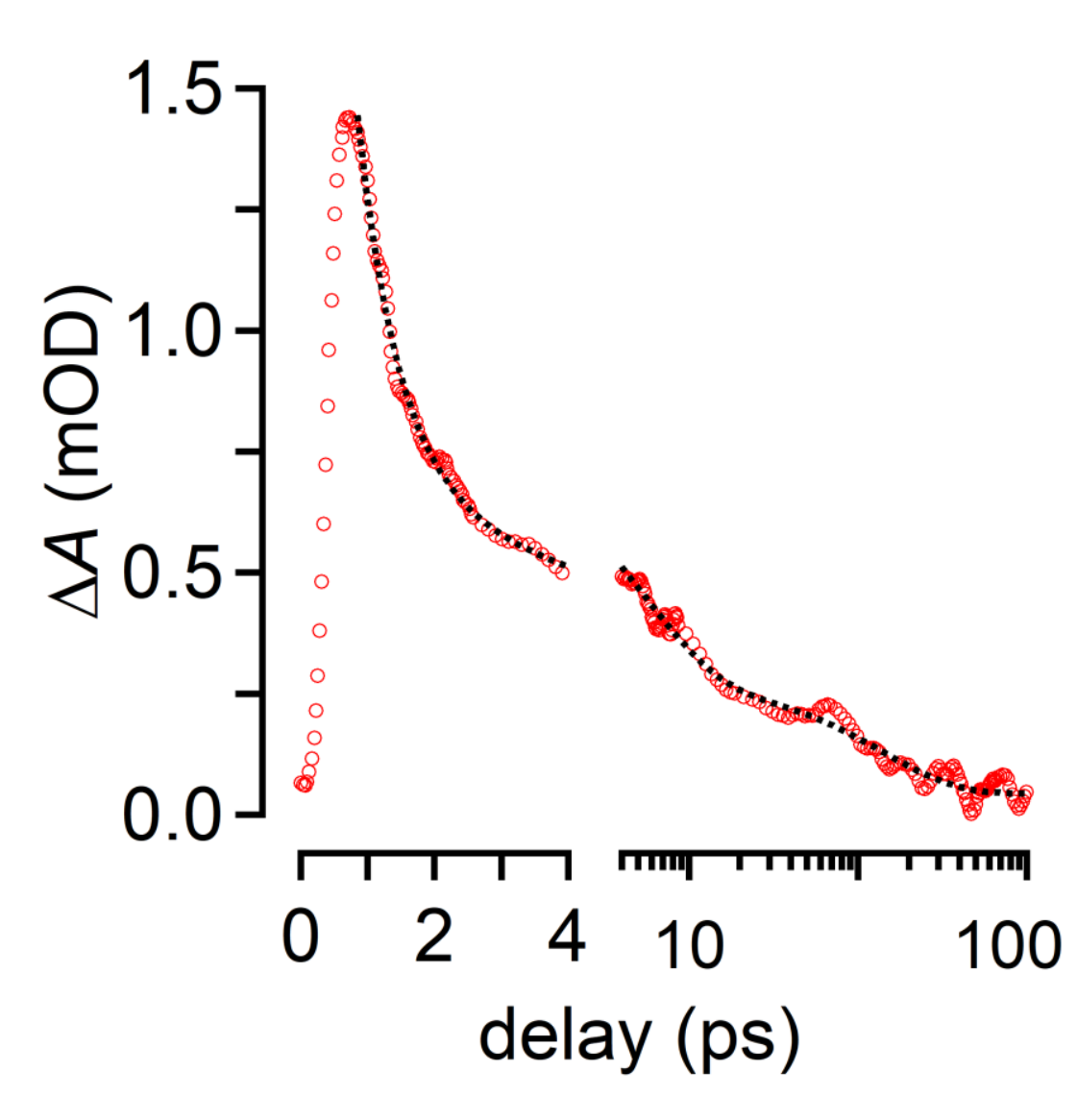

similar dynamics above gap and sub-gap

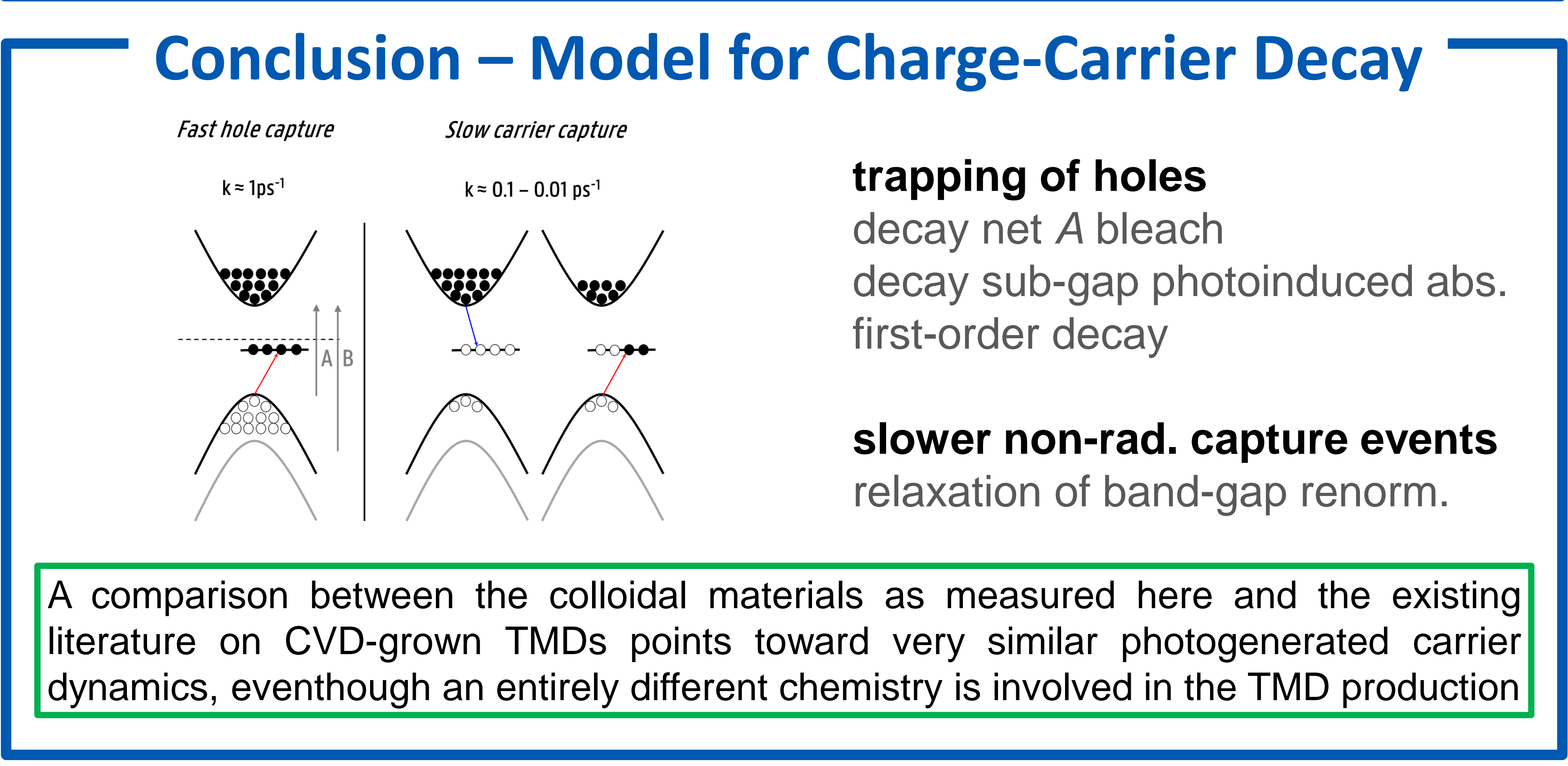

